УДК 616.12-089.168.1-06

DOI 10.17802/2306-1278-2018-7-4-84-91

\title{
IMMUNOSUPPRESSION AS A COMPONENT OF MULTIPLE ORGAN DYSFUNCTION SYNDROME FOLLOWING CARDIAC SURGERY
}

\author{
E.V. Grigoryev ${ }^{1,2}$, D.L. Shukevich ${ }^{1,2}$, V.G. Matveeva ${ }^{1}$, R.A. Kornelyuk ${ }^{1,2} \bowtie$
}

${ }^{I}$ Federal State Budgetary Institution "Research Institute for Complex Issues of Cardiovascular Diseases", 6, Sosnoviy Blvd., Kemerovo, 650002, Russian Federation; ${ }^{2}$ Federal State Budgetary Educational Institution of Higher Education "Kemerovo State Medical University" of the Ministry of Healthcare of the Russian Federation, 22a, Voroshilova St., Kemerovo, 650029, Russia

\section{Highlights}

- The immediate postoperative period after open heart surgeries with extracorporeal circulation is accompanied by immunosupression developing independently of the presence or absences of postoperative complications.

- Elevated levels of monocytic myeloid-derived suppressor cells and IL-10, an anti-inflammatory cytokine, are associated with a complicated postoperative period and persistence of multiple organ dysfunction syndrome.

Aim

Methods

Results

Conclusion

Keywords
To define the role of myeloid-derived suppressor cells in the development of persistent multiple organ dysfunction followed cardiac surgeries with cardiopulmonary bypass.

40 patients who have undergone cardiac surgery were included in the study. Granulocyte myeloid-derived suppressor cells (G-MDSC) were defined as cells with the HLA-DR $-/$ CD $11 \beta^{+} / \mathrm{CD}^{-} 5^{+} / \mathrm{CD}^{+} 3^{+}$phenotype, and monocytic MDSC (M-MDSC) as cells with the HLA-DR ${ }^{-} / \mathrm{CD}_{11} \beta^{+} / \mathrm{CD}^{+} 4^{+} / \mathrm{CD}^{3} 3^{+}$phenotype using flow cytometry. Levels of cytokines, IL-1 $\beta$, IL-6, TNF- $\alpha$, and IL-10 were measured with an enzyme immunoassay. All patients were assigned to three groups: Group 1 - patients with the uncomplicated postoperative period $(n=14)$, Group 2 - patients with non-persistent MODS and its early resolution $(n=16)$, and Group 3 - patients with persistent MODS at day 7.

We observed an increase in M-MDSCs and G-MDSC at day 1 following cardiac surgery. The most pronounced increase was found in monocytic-myeloid derived suppressor cells, i.e. an 8-fold increase in M-MDSCs in all study groups at day 1 after surgery. The number of M-MDSCs remained high in patients with persistent MODS at day 7 after cardiac surgery. Levels of IL-6 and IL-10 increased at day 1 after surgery. IL-6 reached its peak level, significantly exceeding baseline levels. By day 7, blood levels of all cytokines have decreased, except IL-10 levels, which remained above the baseline in patients with persistent MODS.

An increase in M-MDSCs and elevated serum levels of the anti-inflammatory cytokine IL-10 have been found in patients regardless of the presence or absence of the complications in the early postoperative period after cardiac surgery with cardiopulmonary bypass. Persistent MODS with the SOFA scoring $>5$ scores at day 7 after cardiac surgery, is associated with an increase in M-MDSCs and elevated levels of the anti-inflammatory cytokine IL-10, related to higher rate of hospital infections, prolonged intensive care unit stay and higher mortality.

Systemic inflammatory response - Cardiac surgery • Cardiopulmonary bypass • Persistent multiple organ dysfunction syndrome $\bullet$ Myeloid-derived suppressor cells

Received: 07.08.18; received in revised form: 09.09.18; accepted: 07.11.18

\section{Список сокращений}

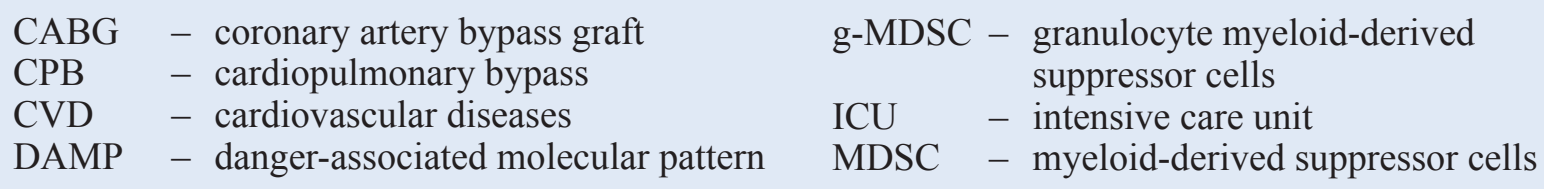




$\begin{array}{rlrl}\text { MODS } & - \text { multiple organ dysfunction syndrome } & \text { SIRS }- & \text { systemic inflammatory response } \\ \text { mon-MDSC }- & \text { monocytic myeloid-derived } & \text { syndrome } \\ & \text { suppressor cells }\end{array}$

\section{Background}

According to the WHO, of the 56.4 million deaths worldwide in 2015 , more than half $(54 \%)$ were due to cardiovascular diseases (CVD) (coronary artery disease and stroke) [1]. These diseases remain the leading causes of deaths in the last 15 years. Treatment for coronary artery disease depends on severity. In selected patients, surgery or other procedures might be needed (isolated coronary artery bypass graft (CABG) or combined with endovascular procedures or heart valve repair). However, a target of $300 \mathrm{CABG}$ procedures per million adult population was set in Russia. But, mainly regions with direct access to cardiac surgery conform to 200 CABG operations [2]. Despite all recent advances in optimizing myocardial protection, cardiopulmonary bypass, and the use of minimally invasive approaches, heart surgeries still require extracorporeal circulation, myocardial protection from injury during aortic crossclamping and cardioplegic cardiac arrest by injecting cardioplegic solutions in the coronary bed. It leads to myocardial ischemia-reperfusion injury which remains the primary cause of acute heart failure during the restoration of spontaneous circulation and results in the onset of post-perfusion multiple organ dysfunction syndrome (MODS) [3-6]. The presence of comorbidities may also complicate the course of the postoperative period in patients with CVD following surgical repair. One theory is that comorbidities are associated with chronic organ dysfunctions, which significantly worsens the process of patient recovery and counteract the regression of MODS [7-11].

Systemic inflammation and, as a consequence, the genetic determination of the so-called host response, can regress even in the presence of the significant clinical risk factors (shock, prolonged ischemia and reperfusion, comorbidities) [12, 13]. The systemic inflammatory response syndrome (SIRS) in the pathogenesis of sepsis has been studied well, whereas sterile inflammation (a pathological condition caused by ischemia-reperfusion injury during on-pump surgeries, severe combined trauma, cardiogenic shock) remains a source of discussion. One of the most significant mechanism, requiring particular attention, is the relationship between the host response and sterile factors initiating the SIRS [14-17].

Immunosuppression is actively involved in the development of multiple organ dysfunction in critically ill patients with prolonged ICI stay. There is a fairly extensive evidence-based experimental and clinical data suggesting immunosuppression to play a key role in the genesis of long-term MI [18-20].

The recent experimental and clinical trials focused on the growth and development of tumors determined a new population of immature myeloid cells with immunosuppressive properties, which were called myeloid-derived suppressor cells (MDSC) [21, 22]. Most of the clinical trials studied the role of MDSC in the development of tumors, reporting the presence of immune suppressive effects of this cell population [23]. However, recent studies demonstrated that the role of MDSC is not limited to cancer patients, but might have beneficial effects on any chronic or acute inflammation $[24,25]$.

Extracorporeal circulation is an ideal model of the systemic inflammatory response due to nonphysiological activation of tissue factors during extracorporeal perfusion, the use of non-pulsatile flow during CPB, intentional / unintentional hypothermia, bacterial translocation from the gut following perfusion deficit and activation of danger-associated molecular patterns (DAMPs) [26, 27]. Monocyte cells during uncomplicated $\mathrm{CPB}$ show their suppressor function, which may be a predictor of $\mathrm{CPB}$-associated complications.

Therefore, the aim of our study was to determine the role of myeloid supressor cells in the development of persistent multiple organ dysfunction syndrome following cardiac surgery with CPB.

\section{Study population}

An observational prospective study was initiated as a part of the project "Myeloid-Derived Suppressor Cells in Cardiac Surgery Patients (MyDeCCS, NCT 02902939). 40 patients admitted to the ICU after elective cardiac surgery with CPB from September 2016 to March 2017 were included in the study (Table 1). All the patients were older than 18 years old. We excluded from the study patients with estimated life expectancy of fewer than 48 hours, those who were transferred to the ICU on corticosteroids and other hormonal drugs, and those who received permanent immunosuppressive therapy and were treated for acute coronary syndrome. Patients did not sign informed consents because the study was observational.

All the patients were divided into three groups: Group 1 - patients with the uncomplicated postoperative course $(n=14)$, Group 2 - patients who had non- 
persistent MODS with symptom resolution in the postoperative period $(\mathrm{n}=16)$, and Group 3 - patients with persistent MODS without symptom resolution $(\mathrm{n}=10)$. Group assignment principle was used as in [14]. Group 2 patients with MODS, which resolved at day 7, demonstrated a decrease in the SOFA scores ( $<5$ scores), whereas Group 3 patients with persistent MODS had $>5$ SOFA scores.

\section{Standard treatment protocol}

All patients underwent cardiac surgery (coronary artery bypass grafting or heart valve repair or combined surgeries; details of the performed cardiac procedures are presented in Table) with CPB using the heart-lung machines (Terumo System1, Jostra HL20, Japan) and oxygenators (Eurosets, Italy). Extracorporeal circulation was performed using non-pulsatile (normothermia $-80 \%$ of patients, $20 \%$ - superficial hypothermia) flow with the calculated perfusion index of $2.5-2.7 \mathrm{1} / \mathrm{min} / \mathrm{m} 2$. Cold blood cardioplegia was used in $78 \%$ of patients according to the standard perfusion protocol. Custodiol cardioplegic solution (Dr. F. Kohler Chemie, GmbH, Germany) was used in patients undergoing heart valve repair. The optimal method of cardioplegia delivery was confirmed together with the surgeon. Local standard hemodynamic and laboratory monitoring, including Swan-Ganz catheter, calculation of oxygen delivery and consumption, and blood gas analysis, was performed.

Anesthesia: endotracheal anesthesia, induction, and maintenance - total i.v. anesthesia with propofol at dose rate of $0.1-0.3 \mathrm{mg} / \mathrm{kg} / \mathrm{min}$ (Propofol Lipuro, BBBrown, Germany) and fentanyl at dose rate of 1-3 $\mu \mathrm{g} / \mathrm{kg} / \mathrm{h}$, myoplegia with atracurium ("Tracrium", Wellcome Foundation, Ltd., Great Britain) at dose rate of $0.3-0.6 \mathrm{mg} / \mathrm{kg} / \mathrm{h}$. The adequacy of anesthesia was assessed according to the level of BIS.

\section{Phenotype analysis}

Peripheral venous blood was collected into a sterile K3-EDTA VACUTAINER blood collection tube before the surgery (point 0 ), at day 1 (point 1), at day 2 (point 2) and at day 7 (point 3) after cardiac surgery. Cytofluorimetric analysis was performed using a four-channel flow cytometer FACS Calibur (Becton Dickinson, USA). All samples were prepared according to the manufacturer's protocol. HLA-DR monoclonal antibodies conjugated to FITC (BC), CD11ß-PE, CD15-PerCP, CD14-PerCP and CD33APC (BioLegend, USA) were used. $100 \mu$ of whole blood with monoclonal antibodies were incubated for 30 minutes in the dark at room temperature. Red blood cells were lysed using diluted BD lysing solution (Becton Dickinson, USA). Then, all the samples were

Table. Baseline characteristics of the study population in the preoperative period, Me (min-max)

\begin{tabular}{|c|c|c|c|}
\hline Parameter & $\begin{array}{l}\text { Patients with the } \\
\text { uncomplicated } \\
\text { postoperative course } \\
\text { (Group } 1, \mathbf{n}=14 \text { ) }\end{array}$ & $\begin{array}{c}\text { Patients with MODS } \\
\text { regression } \\
\text { (Group } 2, n=16)\end{array}$ & $\begin{array}{c}\text { Patients with } \\
\text { persistent MODS } \\
\text { (Group 3, } n=10 \text { ) }\end{array}$ \\
\hline Age, years & $57.2(42.0-66.0)$ & $59.2(48.0-66.9)$ & $67.2(49.0-77.0)$ \\
\hline Male, $\%$ & $14(100)$ & $15.0(93.75)$ & $8(80)$ \\
\hline Body mass index, $\mathrm{kg} / \mathrm{m} 2$ & $33.4(32.1-35.2)$ & $36.4(32.0-36.7)$ & $31.4(31.1-33.2)$ \\
\hline Comorbidity index, CIRS, scores & $9.6(8.0-11.1)$ & $9.0(8.9-11.5)$ & $10.8(9.9-12.1)$ \\
\hline CABG, $\%$ & $8(57)$ & $8(50)$ & $5(50)$ \\
\hline Heart valve repair \% & $2(14)$ & $4(25)$ & $3(30)$ \\
\hline Combined surgeries, $\%$ & $4(29)$ & $4(25)$ & $2(20)$ \\
\hline $\begin{array}{l}\text { MODS causes: } \\
\text { - low cardiac output syndrome } \\
\text { - acute massive blood loss } \\
\text { - combination }\end{array}$ & N/A & $\begin{array}{l}8(50) \\
8(50) \\
0\end{array}$ & $\begin{array}{l}4(40) \\
3(30) \\
3(30)\end{array}$ \\
\hline Renal replacement therapy sessions per patient & 0 & $0.5(0-1.2)$ & $2.3(2.1-4.3)^{* *} / \#$ \\
\hline Initial APACHE II scoring, scores & $1.2(1.0-3.4)$ & $10.9(9.7-13.5)^{*}$ & $26.6(19.0-28.9)^{* *} / \#$ \\
\hline $\begin{array}{l}\text { Initial SOFA scoring at admission to the ICU, } \\
\text { scores }\end{array}$ & $0.9(0-1.9)$ & $5.6(5.1-7.8)^{*}$ & $7.8(7.1-12.0)^{* *}$ \\
\hline ICU stay, days & $3(1-4)$ & $11(10-14)^{*}$ & $21(19-33) * / * * / \#$ \\
\hline Postoperative infections, $\%$ & 0 & $10(62.5)$ & $10(100)$ \\
\hline 28-day mortality, \% & 0 & 9 & 18 \\
\hline
\end{tabular}

Note: *-N/A - not applicable; $p<0.05$ when comparing Groups 1 and 2, **-p<0.05 when comparing npu Groups 1 u 3, \#-p<0.05 when comparing Groups 2 and 3 . 
washed in phosphate buffered saline (PBS) of high concentration. The HLA-DR / CD11 $\beta$ / CD15 / CD33 panel was used to detect G-MDSC, and the HLA-DR / CD14 / CD33 panel - M-MDSC. Flow cytometer parameters were the same for all samples. M-MDSC population was gated on an SS / CD14 bivariate histogram, and G-MDSC population - on an SS / CD15 plot. G-MDSC were defined as cells with the HLA-DR ${ }^{-}$ / CD $11 \beta^{+} / \mathrm{CD} 15^{+} / \mathrm{CD} 3^{+}$phenotype, and M-MDSC as cells with the HLA-DR ${ }^{-} / \mathrm{CD}_{11} \beta^{+} / \mathrm{CD} 4^{+} / \mathrm{CD} 3^{+}$ phenotype.

Level of cytokines, IL-1 $\beta$, IL-6, TNF- $\alpha$ and IL10 , were measured by an enzyme immunoassay using the manufacturer's protocol (Bender Medsystems, Germany).

The total peripheral white blood cells count was computed in the standardized clinical diagnostic laboratory of the Research Institute.

\section{Statistical analysis}

The mean and median were calculated using a GraphPad Prism Software Version 6 (GraphPad Software, Inc., USA). The Kruskal-Wallis test and the t-test with the Bonferroni-Dunn correction were used for multiple comparisons.

\section{Results}

According to the baseline data (Table 1), patients with persistent MODS and non-persistent MODS, resolved within several days, had similar age, gender and the number of comorbidities. However, patients, included in Groups 2 and 3, had significantly higher APACHE II scores, compared to Group 1 patients. The between-group comparison reported that combined surgeries prevailed in Groups 2 and 3, compared to Group 1 patients. However, this difference did not quite reach statistical significance. Low cardiac output syndrome was the most common cause of the postoperative MODS onset. Group 3 patients with persistent MODS had significantly higher SOFA scores and the number of renal replacement therapy sessions, performed according to the presence of renal and non-renal indications. Groups 2 and 3 patients had significantly prolonged ICU stay, compared to Group 1 patients. Group 3 patients had significantly higher rate of infections of various locations, compared to Group 2 patients with non-persistent MODS. No cases of infections detected in Group 1 patients.

Serial changes in the number of MDSCs are presented in Figures 1 and 2 (G- and M-MDSC, respectively).

At day 1 after cardiac surgery with cardiopulmonary bypass, the number of M- and G-MDSCs increased in all study groups. The following trend in G-MDSCs has been found: the increase in their number was detected after the surgery, but the between-group comparison did not report any statistical difference. It should be

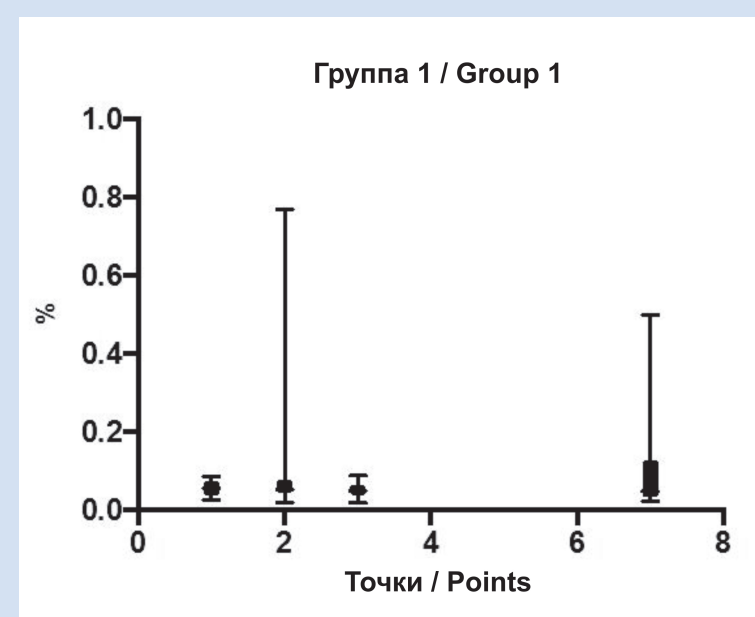

Группа 2 / Group 2

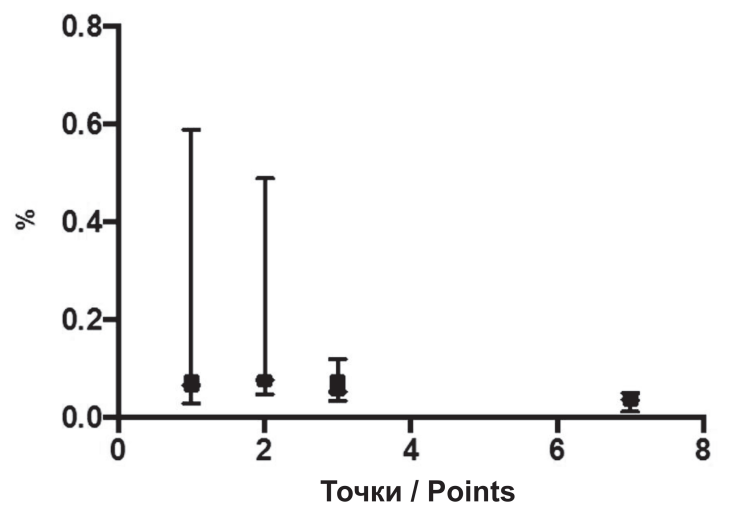

Группа 3 / Group 3

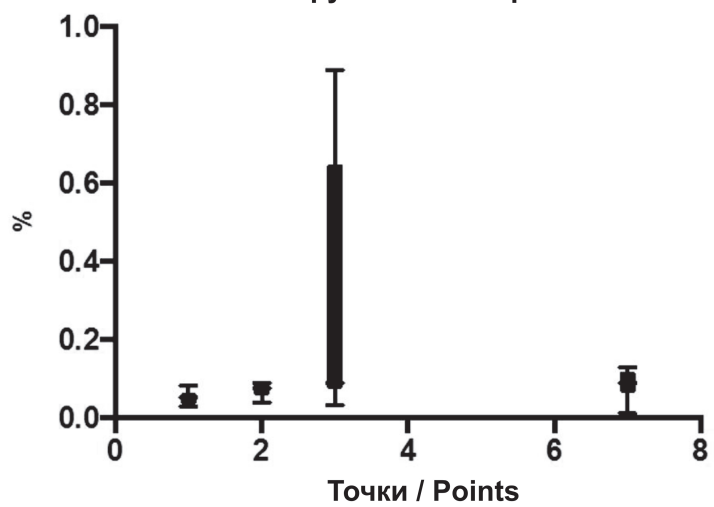

Figure 1. Serial changes in the number of G-MDSC in the study groups

Note: Group 1 - patients with the uncomplicated postoperative course. Group 2 - patients with MODS and its regression at day 7 (the SOFA scoring < 5 scores). Group 3 - patients with persistent MODS at day 5 (the SOFA scoring > 5 scores). Points: 1 - baseline values, 2-day 1, 3-day 2, 4 -day 7 (for all plots). The Y-axis-G-MDSC, \%.

noted that the portion of G-MDSCs was higher in patients with persistent MODS at day 3, compared to the other study groups. However, the data scattering did not allow us confirming the reliability of the abovedescribed differences.

The most pronounced increase was observed for monocytic-myeloid derived suppressor cells, i.e. an 8 -fold increase in M-MDSC at day 1 after the surgery (the differences are significant in comparison with the baseline 
levels, but they did not reach statistical significance in the between-group comparison). At day 7, the number of M-MDSCs decreased to baseline in patients without MODS. The percentage of M-MDSCs increased in Groups 2 and 3 at day 7 after surgery. The highest level of M-MDSCs was observed in patients with persistent MODS.

At day 1 after cardiac surgery, all cytokine levels increased (Figure 2). The most significant changes were found in the levels of TNF- $\alpha$, IL-6 and IL-10. The level of TNF- $\alpha$ was significantly higher at day 2 postoperatively, compared to that level at day 1 in Groups 1 and 2. At day 7, it decreased to the values, which are comparable with the comparison groups. IL-6 achieved its peak level at day 2 after cardiac surgery, compared to that at day 1 . At day 7 , blood levels of cytokines decreased.

Elevated levels of IL-10 were observed in patients with the complicated postoperative course and those with persistent MODS, compared to baseline levels and according to the between-group analysis.

\section{Discussion}

According to the obtained results, we concluded that the use of standard clinical and laboratory criteria for systemic inflammatory response in cardiac surgery may be misleading, if diagnostic criteria of the SIRS are extrapolated from the R. Bone's sepsis classification without considering the sensitivity and specificity of the latter with respect to $\mathrm{CPB}$, cardioplegic arrest and peculiarities of cardiac surgeries (i.e. hypothermia, nonpulsatile flow, hemostatic derangement, etc.) [28, 29]. Along with other researchers, we have demonstrated the need to improve laboratory instruments for the SIRS criteria, particularly with regard to the possibility of introducing parameters for assessing innate immunity [30].

The results obtained in the previous studies demonstrate that blood levels of all cytokines (IL-1 $\beta$, IL-6, IL-10, and TNF- $\alpha$ ), particularly IL-6 and IL-10, increase significantly at day 1 following cardiac surgery with CPB. The early postoperative period in cardiac patients undergoing surgery with $\mathrm{CPB}$ is commonly characterized by the onset of SIRS of varying severity, accompanied by hypercytokinemia [31,32].

Currently, it is quite clear that in critically ill patients, who have trauma, sepsis, etc., inflammation and immune suppression occur almost simultaneously for a long-term period. Mortality rate in critical patients with MODS decreased to $23-25 \%$, but remains at this level due to the effects of persistent inflammation, which is known as catabolic MODS. Moore et al. [20] introduced the term persistent inflammation, immunosuppression, and catabolism syndrome (PICS) to cover this critical state.

One of the hypotheses suggests that immunosuppression is induced in patients without comorbidities and during uncomplicated $\mathrm{CPB}$ with

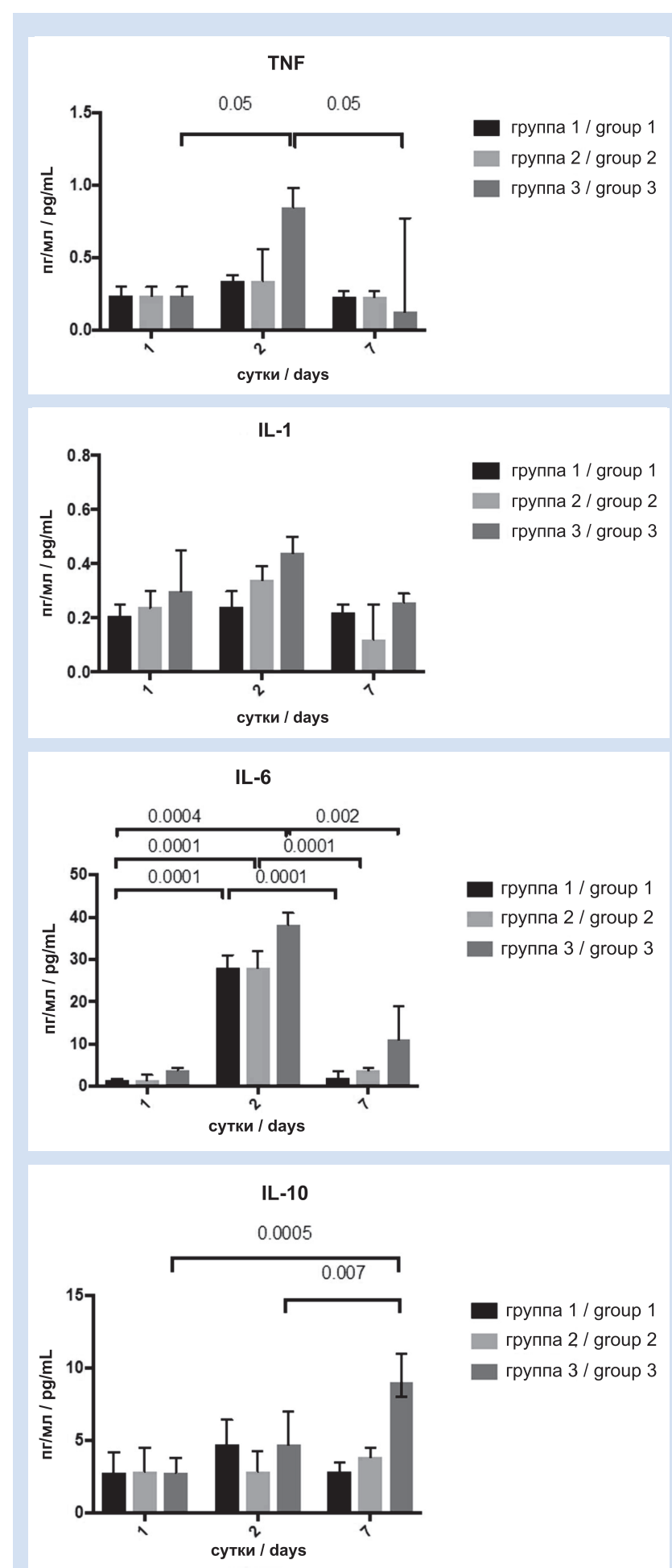

Figure 2. Serial changes in cytokine serum levels in the study groups

Note: Group 1 - patients with the uncomplicated postoperative course. Group 2 - patients with MODS and its regression at day 7 (the SOFA scoring < 5 scores). Group 3 - patients with persistent MODS at day 5 (the SOFA scoring > 5 scores). Points: day 1, day 2, day 7 (for all plots).

an average duration of the aortic cross-clamping. This concept is confirmed by an increase in the expression of anti-inflammatory cytokines and a decrease in HLADR expression [33]. A number of studies have proven that immune suppression appears to be a basis for the onset of persistent MODS, but the exact underlying mechanisms of the latter are still unclear. Clinical, laboratory, immunological and genetic markers have 
already been identified, but monitoring parameters require further clarification [34].

Extreme myelopoiesis has been reported to result in the expansion of myeloid-derived suppressor cells to the bone marrow, secondary lymphoid organs with the reticuloendothelial system [35]. Myeloid-derived suppressor cells are a heterogeneous cell population and may potently suppress $\mathrm{T}$ cell functions due to the production of iNOS, arginase and reactive oxygen intermediate (ROI) [36]. The activity of such cells directly correlates with immune suppression [37-39]. This role is significant and may be a key one in the development of persistent inflammation. These cells produce a large number of cytokines (IL-10, TNF- $\alpha$, etc) in addition to ROI and nitric oxide, which have immunosuppressive effects [40].

IL-10 is of primary importance among other antiinflammatory cytokines. Il-10 reaches its peak blood concentration within 2 hours after the SIRS onset in patients who have undergone cardiac surgery with $\mathrm{CPB}$. It starts decreasing at day 2 [41, 42]. IL-10 inhibits the production of pro-inflammatory cytokines such as IL- 6 and TNF- $\alpha$ by macrophages and monocytes, reduces the expression of major histocompatibility complex molecules, and bone stimulatory growth factors, etc. Recent studies have reported the presence of the relationship between blood levels of IL-6, IL-10 and the risk of infection in the postoperative period, which is associated with the development of an immunosuppressive phenotype after CPB. We noted that anti-inflammatory response in the late postoperative period prevailed in cardiac patients included in our study due to high levels of IL- 10 .

MDSCs, obtained from tumor-bearing experimental animals and / or cancer patients, are known to produce an increased amount of the anti-inflammatory and immunosuppressive cytokine IL-10. Therefore, we may assume that MDSCs may potentiate IL-10-dependent immune suppression and polarization of T-helpers, as well as stimulate the formation of regulatory $\mathrm{T}$ cells [43]. We obtained similar data in our study. The expression of M-MDSC increased at day 1 following cardiac surgery with $\mathrm{CPB}$, accompanied by elevated serum levels of IL-10. However, elevated levels of proinflammatory IL-6 are also of paramount importance, as they confirm the presence of the balanced systemic inflammatory response to CPB [26].

The role of MDSCs in the development of sterile systemic inflammatory response requires further investigation. Currently, there is lack of studies as we found only one study focused on the involvement of
M-MDSCs in the development of infectious pulmonary complications following surgeries with CPB $[33,44]$.

We confirmed in a relatively small cohort of patients that prolonged MODS with the SOFA scoring $>5$ scores is associated with higher proportion of cells with the HLA-DR ${ }^{-} / \mathrm{CD} 11 \beta^{+} / \mathrm{CD} 14^{+} / \mathrm{CD} 33^{+}$ phenotype and elevated levels of anti-inflammatory cytokine, IL-10, related to immune suppression. During the inflammation, the release of MDSC and analogues increases dramatically due to both DAMPs and PAMPs (pathogen-associated molecular patterns) activity, leading to the extreme reorganization of myelopoiesis. Myeloid cells play important role in the realization of the innate immune response, in the release of systemic inflammatory mediators and in the activation of the acquired immune response [45]. Normally, the physiological quantity of mature neutrophils and monocytes is achieved due to uniform myelopoiesis. In acute inflammation, mature neutrophils and less mature cells mobilized from blood and bone marrow are guided to the exact location of the inflammatory focus with underlying systemic endotheliosis and SIRS-induced vasculitis [31]. The result of this response is the rapid depletion of bone marrow reserves and the ejection of local mediators, causing "urgent" myelopoiesis.

\section{Conclusion}

An increase in M-MDSCs and elevated serum levels of the anti-inflammatory cytokine IL-10 have been found in patients regardless of the presence or absence of the complications in the early postoperative period after cardiac surgery with cardiopulmonary bypass. Persistent MODS with the SOFA scoring > 5 scores at day 7 after cardiac surgery, is associated with an increase in M-MDSCs and elevated levels of the anti-inflammatory cytokine IL-10, related to higher rate of hospital infections, prolonged ICU stay and higher mortality.

\section{Conflict of interest}

E.V. Grigoryev declares that there are no conflicts of interest related to this article. D.L. Shukevich declares that there are no conflicts of interest related to this article. V.G. Matveeva declares that there are no conflicts of interest related to this article. R.A. Kornekyuk declares that there are no conflicts of interest related to this article.

\section{Funding}

The authors declare that there is no funding related to this article.

\section{Author Information Form}

Grigoriev Evgeny V., MD, PhD, Professor of the Russian Academy of Sciences, Deputy Director for Research and Clinical Issues, Federal State Budgetary Institution "Research Institute for Complex Issues of Cardiovascular Diseases", Kemerovo, Russian Federation; Head of the Department of Intensive Care, Federal State Budgetary Educational Institution of Higher Education "Kemerovo State Medical University" of the Ministry of Healthcare of the Russian Federation; 
Shukevich Dmitriy L., MD, PhD, Head of the Laboratory of Critical Conditions, Federal State Budgetary Institution "Research Institute for Complex Issues of Cardiovascular Diseases", Kemerovo, Russian Federation; Professor at the Department of Intensive Care, Federal State Budgetary Educational Institution of Higher Education "Kemerovo State Medical University" of the Ministry of Healthcare of the Russian Federation;

Matveeva Vera G., PhD, senior researcher at the Laboratory of Cell Technologies, Federal State Budgetary Institution "Research Institute for Complex Issues of Cardiovascular Diseases", Kemerovo, Russian Federation;

Kornelyuk Roman A., MD, intensivist, PhD student, research assistant, Federal State Budgetary Institution "Research Institute for Complex Issues of Cardiovascular Diseases", Kemerovo, Russian Federation; lecturer assistant at the Department of Intensive Care, Federal State Budgetary Educational Institution of Higher Education "Kemerovo State Medical University" of the Ministry of Healthcare of the Russian Federation.

\section{Author Contribution Statement}

$G E V$ - data interpretation, manuscript writing, editing, approval of the final version, fully responsible for the content; $S D L$ - data interpretation, manuscript writing, editing, approval of the final version, fully responsible for the content; $M V G$ - data interpretation, manuscript writing, editing, approval of the final version, fully responsible for the content; $K R A$ - data interpretation, manuscript writing, editing, approval of the final version, fully responsible for the content.

\section{REFERENCES}

1. Mortality and global health estimates. Available at: http://www.who.int/gho/mortality burden disease/en/ (accessed 9.10.2018)

2. Kolh P., Windecker S., Alfonso F., Collet J.P., Cremer J., Falk V. et al. 2014 ESC/EACTS guidelines on myocardial revascularization. Eur J Cardiothorac Surg. 2014;46:517-592. DOI 10.1093/ejcts/ezu366

3. Calvano S.E., Xiao W., Richards D.R., Felciano R.M., Baker H.V., Cho R.J. et al. A network-based analysis of systemic inflammation in humans. Nature. 2005;437:1032-1037. DOI 10.1038/nature 03985

4. Stoppelkamp S., Veseli K., Stang K., Schlensak C., Wendel H.P., Walker T. Identifi-cation of Predictive Early Biomarkers for Sterile-SIRS after Cardiovascular Surgery. PLoS ONE. 2015;10(8): e0135527. DOI 10.1371/journal. pone. 0135527

5. De Somer F. Recent advances in the comprehension and the management of periop-erative systemic host response during cardiopulmonary bypass. Recent patents on cardiovascular drug discovery. 2012;7(3):180-185. http://dx.doi. org/10.2174/157489012803832865

6. Bianchi M.E. DAMPs, PAMPs and alarmins: all we need to know about danger. Journal of Leukocyte Biology. 2007;81(1):1-5. DOI 10.1189/jlb.0306164

7. MacCallum N.S., Finney S.J., Gordon S.E., Quinlan G.J., Evans T.W. Modified criteria for the systemic inflammatory response syndrome improves their utility following cardiac surgery. Chest. 2014;145(6):1197-1203. DOI 10.1378/ chest.14-0438

8. Boomer J.S., To K., Chang K.C. Immunosuppression in patients who die of sepsis and multiple organ failure. Journal of the American Medical Association. 2011;306(23):2594-2605. DOI 10.1001/jama.2011.1829

9. Nelson J.E., Cox C.E., Hope A.A., Carson S.S. Chronic critical illness. Am J Respir Crit Care Med. 2010;182:446-454. DOI 10.1164/rccm.201002-0210CI

10. Docking R.I., Mackay A., Williams C., Lewsey J., Kinsella J., Booth M.G. Comorbidity and intensive care outcome - a multivariable analysis. Journal of Intensive Care Society, 2014;3(15):205-212. DOI 10.1177/175114371401500306

11. Probst C., Zelle B.A., Sittaro N.A., Lohse R., Krettek C., Pape H.C. Late death after multiple severe trauma: when does it occur and what are the causes? J Trauma. 2009;66:1212-1217. DOI 10.1097/TA.0b013e318197b97c
12. Chen C.J., Kono H., Golenbock D., Reed G., Akira S., Rock K.L. Identification of a key pathway required for the sterile inflammatory response triggered by dying cells. Nature Med. 2007;13:851-856. DOI 10.1038/nm1603

13. Sugita H., Kinoshita Y., Bab H. The duration of SIRS before organ failure is a significant prognostic factor of sepsis. International Journal of Emergency Medicine 2012,5:44. DOI 10.1186/1865-1380-5-44

14. Dewar D., Moore F.A., Moore E.E., Balogh Z. Postinjury multiple organ failure. Injury. 2009;40:912-918. DOI 10.1016/j. injury.2009.05.024

15. Flohé S.B., Flohé S., Schade F.U. Invited review: Deterioration of the immune system after trauma: signals and cellular mechanisms. Innate Immun. 2008;14:333-344. DOI $10.1177 / 1753425908100016$

16. Rankin J.S., Oguntolu O., Binford R.S., Trochtenberg D.S., Muhlbaier L.H., Stratton C.W. Management of immune dysfunction after adult cardiac surgery. J Thorac Cardiovasc Surg. 2011;142:575-580. DOI 10.1016/j.jtcvs.2011.04.042

17. Maier B., Lefering R., Lehnert M., Laurer H.L., Steudel W.I., Neugebauer E.A., Marzi I. Early versus late onset of multiple organ failure is associated with differing patterns of plasma cytokine biomarker expression and outcome after severe trauma. Shock. 2007;28(6):668-674. DOI 10.1097/ shk.0b013e318123e64e

18. Hotchkiss R.S., Monneret G., Payen D. Immunosuppression in sepsis: a novel under-standing of the disorder and a new therapeutic approach. The Lancet Infectious Diseases. 2013;13(3):260-268. DOI 10.1016/S1473-3099(13)70001-X

19. Gentile L.F., Cuenca A.G., Efron P.A., Ang D., Bihorac A., McKinley B.A., Moldawer L.L., Moore F.A. Persistent inflammation and immunosuppression: a common syndrome and new horizon for surgical intensive care. J Trauma Acute Care Surg. 2012;72:1491-1501. DOI 10.1097/TA.0b013e318256e000

20. Dilek N., de Silly R.V., Blancho G., Vanhove B. Myeloid-derived suppressor cells: mechanisms of action and recent advances in their role in transplant tolerance. Frontiers in Immunology. 2012;3:208. DOI 10.3389/fimmu.2012.00208

21. Almand B., Clark J.I., Nikitina E., Beynen J. van, English N.R., Knight S.C., Carbone D.P., Gabrilovich D.I. Increased production of immature myeloid cells in cancer patients: a mechanism of immunosuppression in cancer. Journal of Immunology. 2001;166(1):678-689. DOI 10.4049/ jimmunol.166.1.678 
22. Gabrilovich D.I., Nagaraj S. Myeloid-derived suppressor cells as regulators of the immune system. Nat Rev Immunol. 2009;9:162-174. DOI 10.1038/nri2506

23. Makarenkova V.P., Bansal V., Matta B.M., Perez L.A., Ochoa J.B. CD11b+/Gr-1+ myeloid suppressor cells cause T cell dysfunction after traumatic stress. J. Immunol. 2006;176(4): 2085-2094. DOI 10.4049/jimmunol.176.4.2085

24. Li Q., Pan P-Y, Gu P., Xu D., Chen S-H. Role of immature myeloid Gr-1+ cells in the development of antitumor immunity. Cancer Research. 2004;64(3):1130-1139. DOI 10.1158/00085472.CAN-03-1715

25. Wilhelm W., Grundmann U., Rensing H., Werth M., Langemeyer J., Stracke C., Dhingra D., Bauer M. Monocyte deactivation in severe human sepsis or following cardiopulmonary bypass. Shock. 2002;17:354-360. PMID: 12022753

26. Warren O.J., Smith A.J., Alexiou C., Rogers P.L., Jawad N., Vincent C., Darzi A.W., Athanasiou T. The inflammatory response to cardiopulmonary bypass: part 1 - mechanisms of pathogenesis. Journal of cardiothoracic and vascular anaesthesia. 2009;23(2):223-231. DOI 10.1053/j.jvca.2008.08.007

27. Kuvacheva N.V., Morgun A.V., Hilageva N.A., Malinovskaya N.A., Gorina Ya.V., Pogilenkova E.A. et al. Inflamasommas formation: new mechanisms of the intercellular interactions and secretory cell activity. Sibirskoye medicinskoye obozrenye, 2013,5:3-10. (In Russian)

28. Bozza F.A., Salluh J.I., Japiassu A.M., Soares M., Assis E.F., Gomes R.N. Cytokine as markers of disease severity in sepsis: a multiplex analysis. Crit Care. 2007;11(2):R49. DOI $10.1186 / \operatorname{cc} 5783$

29. Matveeva V.G., Golovkin A.S., Grigoryev E.V. Monocytes subpopulations as a prognostic marker of the severe complications after systemic inflammatory response syndrome due to cardiac surgery. Complex Issues of Cardiovascular Diseases. 2014;4:5-12. DOI 10.17802/2306-1278-2014-4. (In Russian)

30. Matsuda N. Alert cell strategy in SIRS-induced vasculitis: sepsis and endothelial cells. Journal of Intensive Care 2016,4:21. DOI 10.1186/s40560-016-0147-2.

31. Pugin J. How tissue injury alarms the immune system and causes a systemic inflammatory response syndrome. Annals of intensive care. 2012;2(1):27. DOI 10.1186/2110-5820-2-27

32. Gaudriot B., Uhel F., Gregorie M., Gacouin A., Biedermann S., Roisne A. et al. Immune dysfunction after cardiac surgery with cardiopulmonary bypass: beneficial effects of maintaining mechanical ventilation. Shock. 2015;44(3):228233. DOI 10.1097/SHK.0000000000000416

33. Cuenca A.G., Moldawer L.L. Myeloid-derived suppressor cells in sepsis: friend or foe? Intensive Care Medicine. 2012;38(6):928-930. DOI 10.1007/s00134-0122575-3

34. Cuenca A.G., Delano M.J., Kelly-Scumpia K.M., Moreno C., Scumpia P.O., LaFace D.M.et al. A paradoxical role for myeloid-derived suppressor cells in sepsis and trauma. Mo-lecular Medicine. 2011;17(3-4):281-292. DOI 10.2119/ molmed.2010.00178

35. Corzo C.A., Cotter M.J., Cheng P. Mechanism regulating reactive oxygen species in tumor-induced myeloid-derived suppressor cells. Journal of Immunology. 2009;182(9):56935701. DOI 10.4049/jimmunol.0900092

36. Ray A., Chakraborty K., Ray P. Immunosuppressive MDSCs induced by TLR signalling during infection and role in resolution of inflammation. Frontiers in Cellular and Infection Microbiology. 2013;3:52. DOI 10.3389/fcimb.2013.00052

37. Takahashi H., Tsuda Y., Kobayashi M., Herndon D.N., Suzuki F. CCL2 as a trigger of manifestations of compensatory anti-inflammatory response syndrome in mice with severe systemic inflammatory response syndrome. Journal of leukocyte biology. 2006;79(4):789-796. DOI 10.1189/jlb.0705372

38. Mathias B., Delmas A.L., Ozrazgat-Baslanti T., Vanzant E.L., Szpila B.E., Mohr A.M. et al. Human Myeloid-derived Suppressor Cells are Associated With Chronic Immune Suppression After Severe Sepsis/Septic Shock // Ann Surg. 2017; 265(4):827-834. DOI: 10.1097/SLA.0000000000001783

39. Nacionales D.C., Szpila B., Ungaro R., Lopez M.C., Zhang J., Gentile L.F. et al A detailed characterization of the dysfunctional immunity and abnormal myelopoiesis induced by severe shock and trauma in the aged. J Immunol. 2015; 195:2396 -2407. DOI 10.4049/jimmunol.1500984

40. Mare T.A., Treacher D.F., Shankar-Hari M., Beale R., Lewis S.M., Chambers D., Brown K.A. The diagnostic and prognostic significance of monitoring blood levels of immature neutrophils in patients with systemic inflammation. Critical Care. 2015;19:57. DOI 10.1186/s13054-015-0778-z

41. Vincent J.L., Sun Q., Dubois M.J. Clinical trials of immunomodulatory therapies in severe sepsis and septic shock. Clin Infect Dis. 2002;34:1084-1093. DOI 10.1086/339549

42. Grigoryev E., Shukevich D., Matveeva V., Radivilko A., Kornelyuk R. Myeloid-derived suppressor cells after cardiac surgery. Clinicheskaya pathophisiologya. 2017;1:74-79. (In Russian)

43. Derive M., Bouazza Y., Alauzet C., Gibot S. Myeloidderived suppressor cells control microbial sepsis. Intensive Care Medicine. 2012;38(6):1040-1049. DOI 10.1155/2014/598654

44. Gey A., Tadie J.M., Caumont-Prim A., Hauw-Berlemont C., Cynober L., Fagon J.Y. et al. Granulocytic myeloid-derived suppressor cells inversely correlate with plasma arginine and overall survival in critically ill patients. Clin Exp Immunol 2015;180(2):280-288. DOI 10.1111/cei.12567

45. Grigoryev E.V., Shukevich D.L., Matveeva V.G., Pugachev S.V., Kameneva E.A., Kornelyuk R.A. Myeloidderived suppressor cells in critical care patients. Cardiovascular pathology and cardiac surgery. 2016.3(20):20-25. DOI 10.21688/1681-3472-2016-3-20-25. (In Russian)

To cite: E.V. Grigoryev, D.L. Shukevich, V.G. Matveeva, R.A. Kornelyuk. Immunosuppression as a component of multiple organ dysfunction syndrome followed cardiac surgery. Complex Issues of Cardiovascular Diseases. $2018 ; 7$ (4): 84-91. DOI: 10.17802/2306-1278-2018-7-4-84-91 